\title{
Development of Radon Gas Sensor To Monitor The Precursors of Earthquake
}

\author{
Mily Jashank ${ }^{1}$, Sureshchandra J. Gupta ${ }^{2}$, Jayalakshmi Nair ${ }^{3}$ \\ ${ }^{1}$ Sr. Lecturer, Information Technology Dept. Thakur Polytechnic Kandivli (E) Mumbai 400101 \\ 2. Adjunct Professor, University Department of Physics, Kalina Mumbai \\ 3. Sr. Lecturer Electronics \&Telecomm Dept. Thakur Polytechnic Kandivli (E) Mumbai 400101
}

\begin{abstract}
Radon gas is colorless, odorless and tasteless and can only be detected by testing. It naturally occurs from traces of uranium in soils and rocks. It results from the decay of trace amounts of uranium, found in most rock and soil. It is measured using special monitors and expressed in units of bequerels per cubic metre $(\mathrm{Bq} / \mathrm{m} 3)$ or picocuries per litre $(\mathrm{pCi} / \mathrm{L})$. The air pressure inside the laboratory is usually lower than in the soil surrounding the foundation [4]. This difference in pressure draws air and other gases, including radon, from the soil into the atmosphere. The Uranium stays in the ground, but radon gas seeps upward and enters buildings. It can be used to predict the arrival of an earthquake. This is because radon is a radioactive gas and it can be traced by detecting alpha particles emitted during the decay of radon and its daughters. It can be achieved by using alpha sensitive solid state nuclear track detectors or some other suitable radon gas detectors.
\end{abstract}

Keywords: - Precursors of Earthquake, Radioactive gases, Seismology, Transducers, Signal conditioner circuit \& The Intelligence.

\section{INTRODUCTION}

Variations in the environmental energy parameters such as relative humidity, temperature, pressure, earth's magnetic field, conductivity of ionosphere, gravitational field etc either natural or man-made has led to calamities such as tsunamis and earthquakes [1]. An earthquake is a violent movement of the rocks in the Earth's crust. They are the result of a sudden release of energy in the Earth's crust. This creates seismic waves, waves of energy that travel through the Earth. There are large earthquakes and small earthquakes. Big earthquakes can take down buildings and cause death and injury. The study of earthquakes is called seismology.

Seismology studies the frequency, type and size of earthquakes over a period of time.

Some research indicates that prior to an earthquake, some strange lights or gases or fires coming from the earth around fault areas which could be considered as precursors. The strange lights could be caused by piezoelectric effects of rocks grinding against each other under great stress. When an earthquake looms, the rock goes through a strange change, producing intense electrical currents [3]. The gases are thought to be escaping gas from pockets deep below the fault zones which can be ignited by those piezoelectric effects. The important four physical parameters which should be monitored continuously and can be considered as precursors are: Seismic Detection (Using Vibration Sensor), Radon Detection (Using Gas Sensor), Ionization Detection (Using Charge Sensor) and Humidity Detection (Using Humidity Sensor).

The release of radon from natural minerals has been known since long but its monitoring has been used recently as a tool for the earthquake prediction, because the distribution of soil-gas radon concentration [4] is closely related to the geological structure, fracture, nature of rocks and distribution of sources. Therefore, surveying of radon concentration can prospect fracture trace, earthquake forecast, environment monitoring, etc.

\section{SEISMOLOGY}

Seismic Waves: Seismic waves are the waves of energy caused by the sudden breaking of rock within the earth or an explosion. They are the energy that travels through the earth and is recorded on seismographs. Large strain energy released during an earthquake travels as seismic waves in all directions through the Earth's layers, reflecting and refracting at each interface. There are several different kinds of seismic waves, and they all move in different ways [3]. The two main types of waves are body waves and surface waves. Body waves can travel through the earth's inner layers, but surface waves can only move along the surface of the earth like ripples on water. Earthquakes radiate seismic energy as both body and surface waves. Body waves consist of Primary waves (P-waves) and Secondary waves (S-waves), and surface waves consist of Love waves and Rayleigh waves [1]. Under P waves the material particles undergo strains along the direction of energy transmission. 


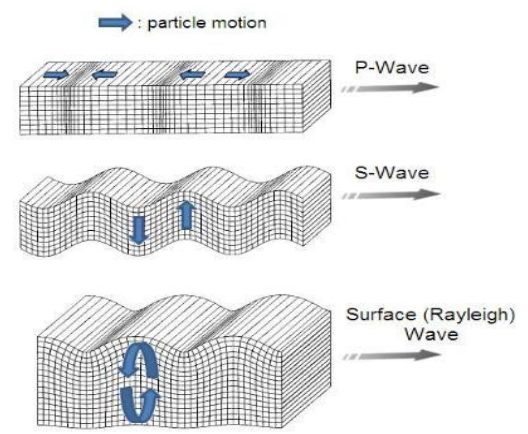

Under $\mathrm{S}$ waves, they oscillate at right angles to it. Love waves cause surface motions similar to that by $\mathrm{S}$ waves, but with no vertical component. Rayleigh wave makes a material particle oscillate in an elliptic path in the vertical plane. $\mathrm{P}$ waves are fastest, followed in sequence by $\mathrm{S}$ waves, Love and Rayleigh waves. In granites $\mathrm{P}$ and $\mathrm{S}$ waves have speeds $\sim 4.8 \mathrm{~km} / \mathrm{sec}$ and $\sim 3.0 \mathrm{~km} / \mathrm{sec}$, respectively. $\mathrm{S}$ waves do not travel through liquids. $\mathrm{S}$ waves in association with effects of Love waves cause maximum damage to structures by their cracking motion on the surface in both vertical and horizontal directions. When $\mathrm{P}$ and $\mathrm{S}$ waves reach the Earth's surface, most of their energy is reflected back. Some of this energy is returned to the surface by reflections at different layers of soil and rock. Shaking is more severe (about twice as much) at the Earth's surface than at substantial depths. This is often the basis for designing structures buried underground for smaller levels of acceleration than those above the ground [1]. Seismology is the study of earthquakes and seismic waves that move through and around the earth. A seismologist is a scientist who studies earthquakes and seismic waves.

A seismograph is the device that scientists use to measure earthquakes [1]. The goal of a seismograph is to accurately record the motion of the ground during a quake.

The Richter scale is a standard scale used to compare earthquakes. It is a logarithmic scale, meaning that the numbers on the scale measure factors of 10 . So, for example, an earthquake that measures 5.0 on the Richter scale is 10 times larger than one that measures 4.0. On the Richter scale, anything below 2.0 is undetectable to a normal person and is called a microquake [3].

\subsection{RADON}

Radon is a colorless, tasteless and odorless radioactive gas produced by the breakdown of uranium in the soil. When allowed to accumulate to high levels, it can be hazardous to long-term health. The Uranium stays in the ground, but radon gas seeps upward and enters buildings. Radon, an inert gas, is produced in the decay series of U-238.

Radon is the main source of radioactivity in earth's atmosphere [3]. It can be used to predict the arrival of an earthquake. This is because radon is a radioactive gas and it can be traced by detecting alpha particles emitted during the decay of radon and its daughters. It can be achieved by using alpha sensitive solid state nuclear track detectors or some other suitable detectors. An important application of radon measurements is in earthquake prediction. The earthquakes have always been a source of terror and destruction for the mankind. The earthquake prediction has always been a dream and now it seems to be at the verge of reality as a result of advances made in the science of radon measurement. Work carried out in this direction was based upon the assumption that significant changes take place in the emission of gases such as radon and trapped in the earth crust before the arrival of a 'physical jolt' of an earthquake.

This change takes place because of the physical stresses which are built up within the earth crust to trigger an earthquake.

Uranium-238 $\left({ }^{238} \mathrm{U}\right.$ or $\left.\mathrm{U}-238\right)$ is the most common isotope of uranium found in nature. Uranium (U) is a metallic, silver-gray element that is a member of the actinide series [3]. The total uranium content of the earth crust is about 3-4 ppm (parts per million) which is significant in terms of total mass and its radiological contribution to our atmosphere. Since Uranium is radioactive, it is constantly emitting particles and changing into other elements. Natural ${ }^{238} \mathrm{U}$ decays into ${ }^{234} \mathrm{Th}$, while the other isotope of $\mathrm{U},{ }^{234} \mathrm{U}$, decays into ${ }^{230} \mathrm{Th}$. The ${ }^{230} \mathrm{Th}$ is itself a radioactive element and it decays into daughter products. The ${ }^{234} \mathrm{Th}$ decays into ${ }^{234} \mathrm{U}$ and the ${ }^{230} \mathrm{Th}$ decays into ${ }^{226} \mathrm{Ra}$. Ra is also radioactive and it decays into Rn in a very short time. 


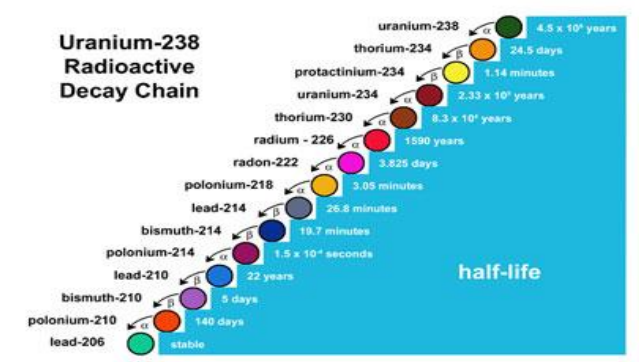

Radioactive decay is the process by which an atomic nucleus of an unstable atom loses energy by emitting ionizing particles (ionizing radiation). There are many different types of radioactive decay [4]. A decay, or loss of energy, results when an atom with one type of nucleus, called the parent radionuclide, transforms to an atom with a nucleus in a different state, or to a different nucleus containing different numbers of protons and neutrons. Either of these products is named the daughter nuclide. In some decays the parent and daughter are different chemical elements, and thus the decay process results in nuclear transmutation (creation of an atom of a new element). The decay processes are alpha decay, beta decay and gamma decay. Alpha decay occurs when the nucleus ejects an alpha particle (helium nucleus). Alpha decay is a type of radioactive decay in which an atomic nucleus emits an alpha particle (two protons and two neutrons) and thereby transforms (or decays) into an atom with a mass number 4 less and atomic number 2 less. This is the most common process of emitting nucleons, but in other two types of decays, nuclei can eject protons, or specific nuclei of other elements (in the process called cluster decay). Beta decay occurs when the nucleus emits an electron or positron and a type of neutrino, in a process that changes a proton to a neutron or the other way around. The nucleus may capture an orbiting electron, converting a proton into a neutron (electron capture). The energy of an excited nucleus may be emitted as a gamma ray in gamma decay, or used to eject an orbital electron by interaction with the excited nucleus in a process called internal conversion. Radioisotopes occasionally emit neutrons, and these results in a change in an element from one isotope to another.

\subsection{DEVELOPMENT OF RADON SENSOR}

The Radon gas sensor consists of two parts i.e. a Radon detector and an ionization chamber to collect the Radon gas. To develop a Radon detector we have to follow the following steps: Cut out one end of a large can with the can opener. Make a hole in the remaining end, as follows: find the center of the bottom of the ionization chamber can. Now, use one of the fingernails pressed against the end of the ruler to establish the radius, and press your fingernail against the can, drawing a few lines from different directions to mark the center. It can be difficult to drill a clean hole in the thin metal, so a satisfactory alternative is to drill a smaller hole and then use a tapered hand reamer to widen the hole to about $1 / 2$-inch diameter. The excess metal will form a short, ragged tube extending into the can, instead of breaking off in bits, but the hole will be smooth and round. Bend the base lead (middle pin) of the two transistors joined together to form the Darlington transistor at a right angle, extending towards the flat side of the body. Solder a piece of solid, 22- or 24-AWG bare wire to the base lead. Cut the bare wire to be shorter than the large can, which will serve as the ionization chamber, by $3 / 4$ inch. Apply a small amount of epoxy to the top end of the transistor, keeping the epoxy well away from the leads. Pass the long wire into the can and press the flat side of the transistor against the bottom of the can (the end with the small hole), allowing the epoxy to spread onto the can. If required we can solder a temporary wire to the can to help hold the transistor while the epoxy cures. Keep a check to make sure the center lead is free from any epoxy. Solder a 1-k resistor in series with the positive (red) battery snap lead and cover the connection with heat shrink tubing or electrical tape. Apply some double-sided foam tape to a narrow side of a 9-V rectangular battery, the side that will result in the wires from the battery snap coming out on top. Stick the battery to the bottom of the ion chamber can, near the hole. Solder the resistor on the positive battery lead directly to the can. Solder the negative battery lead (black) to the emitter of the transistor. Connect a thin, flexible 3-inch wire to the remaining collector lead. Refer the schematic diagram as shown in figure 1 of the completed Radon gas detector. 


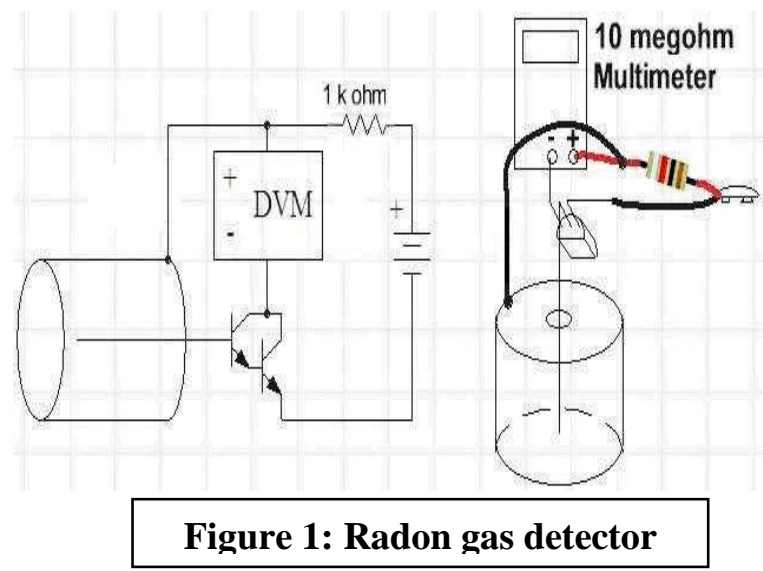

Now to Build a Radon Daughter Collection Device: The ionization chamber is the simplest of all gasfilled radiation detectors, and is widely used for the detection and measurement of certain types of ionizing radiation. Using the can opener, remove both ends of an ordinary soup can, affix the can to the intake of a squirrel cage fan using epoxy. Apply a bead of epoxy to the lip of the can, making sure to leave no gaps, and place the can on the fan's intake. After the epoxy has had plenty of time to cure, cut the connector off the end of the fan's cable with heavy-duty scissors and remove some insulation from the red and black wire with the wire strippers. Cut off the end of the $12-\mathrm{V}$ power supply cable and strip off some of the outer insulation. For the supply positive wire from the supply is connected to the red wire of the fan. The negative wire from the supply goes to the black wire of the fan. The other wires may be cut short. Connect the wires by twisting them together and then soldering. Cover the bare wire with electrical tape or heat-shrink tubing. Add some feet to the bottom of the fan, so that it can stand vertically. Secure a piece of the dusting cloth to the open end of the can with a heavy rubber band. Plug in the fan and verify that it pulls air through the filter. Now the testing of the instrumentation can be done by the following steps: Set up the fan in an area that is likely to have elevated radon, such as a basement. Turn on the fan with the filter facing up and let it run for 2 to 3 hours. Connect the ionization chamber to a digital multimeter and turn on the DC voltage meter. Make an initial meter reading and record it in a lab notebook. Unplug the fan and tip the collector over to the horizontal position. Move the end with the collection cloth as close to the end of the ionization chamber as possible without pressing on the foil with significant force. We find the meter reading increases for several seconds and settle out at a significantly higher reading, indicating that radioactive material has been collected on the collection material. Run the fan in a different room or in other locations for a specific time, perhaps for more hours, and measure the resulting radiation. Different locations are likely to exhibit different levels of radiation due to different levels of radon gas.

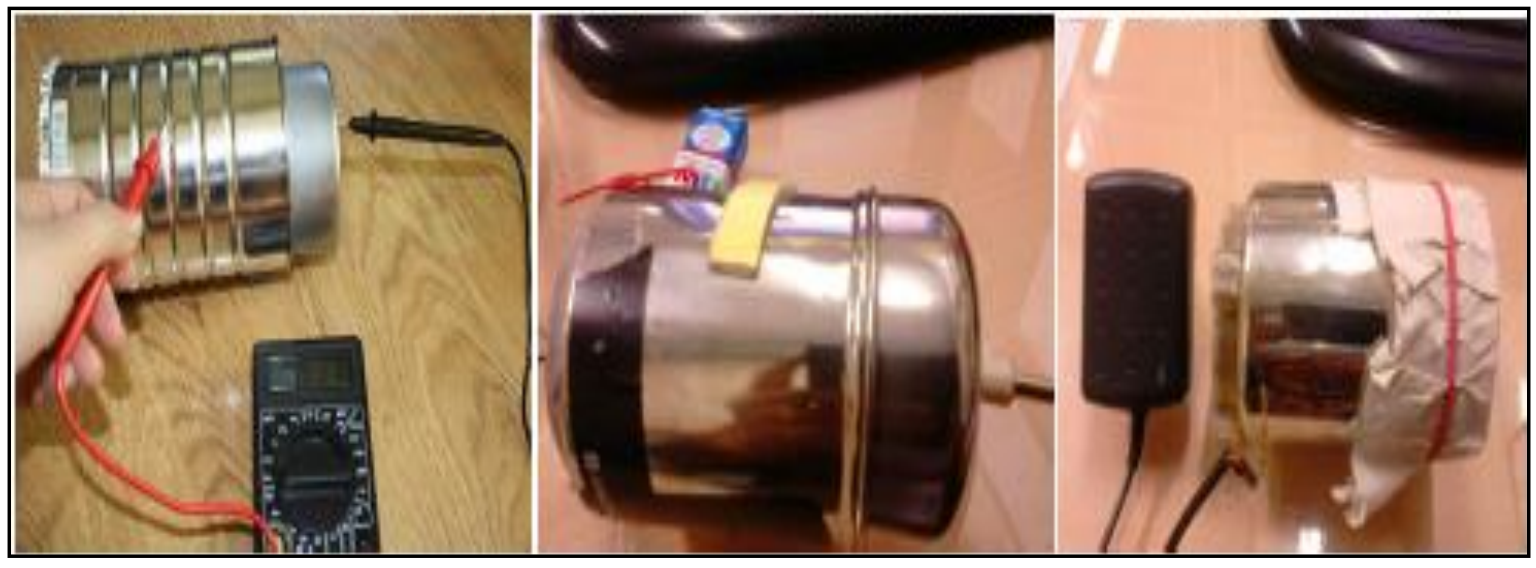

Figure 2: Instrumentation developed for the Ionization chamber and Radon Collector 


\section{INSTRUMENTATION}

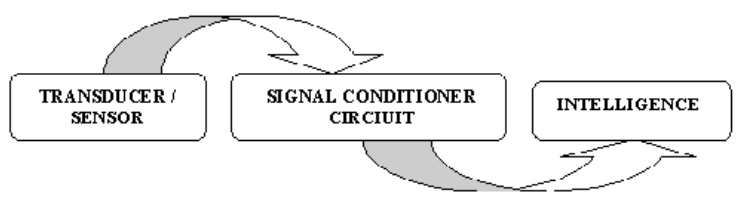

The experimentation system comprises of the following elements: The transducer/sensor which converts the physical parameter into usable electrical output [10]. The signal conditioner which converts the transducer output into an electrical quantity suitable for control, recording and display[9]. The display or read out devices which display the required information about the physical parameter in certain units [10]. The power supply which provides the required excitation to the transducer and the necessary power to the signal conditioners and display devices.

\subsection{TRANSDUCERS/SENSORS}

In order to study control and monitor of any physical parameter through electronic instrumentation we need a device called transducer/sensor. This device converts the variation in the physical parameter to a variable electrical signal. A transducer is defined as a device which when actuated by one form of energy is capable of converting it into another more useful form of energy. The transformation may be from mechanical to electrical, optical or any other form. Transducers usually generate output signals in the $\mathrm{mV}$ range (spans of $10 \mathrm{mV}$ to $250 \mathrm{mV}$ ), often amplified to the voltage level (1 to $5 \mathrm{~V}$ ) and converted to current loops, usually 4-20mA dc [6]. The transducer housing should be selected to meet both the electrical and the corrosion requirements of the particular installation. This is usually achieved either by placing them inside purged or explosion-proof housings or by using intrinsically safe designs.

\subsection{SIGNAL CONDITIONER CIRCUIT}

A signal conditioner can vary in complexity from a simple resistance network or impedance matching network to a complex multistage amplifier with or without detectors, demodulators and filters. Alternately they are termed as signal modifiers or signal processors [6]. The output is usually analog but can be converted into digital using the analog to digital converter. In our experimentation the signal conditioner consists of a buffer (Op-amp as voltage follower), an amplifier (Op-amp in non-inverting mode) with adjustable gain (maximum up to 500), a rectifier followed by a filter (to get smooth dc) and again a buffer (Op-amp as voltage follower) for impedance matching between the signal conditioner circuit and the ADC [10].

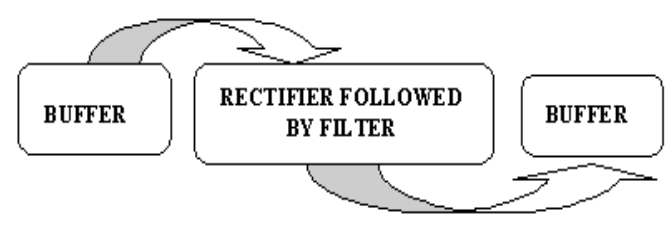

\subsection{THE INTELLIGENCE}

The output of the sensor is interfaced to signal conditioning circuit which in turn is interfaced to an analog to digital converter. The output of the analog to digital converter is an input to either a microprocessor (8085) [6] or a microcontroller (ATmega16) [5] or Microcontroller 89C51 [7] or PIC [8] who exactly monitors and sends warning signal when the parameter exceeds the predefined critical value. The configuration with PIC is most compact compared to all [8].

\section{CONCLUSION}

Hence from the instrumentation part we can understand that by connecting the output of the sensor to a signal conditioning circuit, which amplifies and rectifies it and then to a ADC, which provides a Digital output and finally to an Intelligence which receives the digital output from the signal conditioning circuit and gives the output to the microcontroller or PIC, which can be interfaced with a HyperTerminal or LCD [11] to view the digital output. Therefore by setting a threshold value, if any variations found from the threshold set value, an alarm can be generated to conclude the occurrence of earthquake, quiet before time to avoid major destructions. 


\section{ACKNOWLEDGEMENT}

I would like to express my sincere thanks to my guide Dr. Sheshmani K. Dubey, Sr. Professor, UDP, Dr. Bhatia, Professor ACRET, Dr. D.C. Kala, Professor Khalsa College of Science and Commerce and my entire dear and near ones who have always supported and motivated me for my research work.

\section{REFERENCES}

[1]. Cinna Lomnitz, John Wiley \& Sons Ltd. Fundamentals of earthquake prediction:.

[2]. Friedmann, H., Aric, K., Gutdeutsch, R., King, C.Y., Altay, C., Sav, H. (1988). Radon measurements for earthquake prediction along the North Anatolian Fault Zone: a progress report. Tectonophysics Designing Embedded Systems with PIC Microcontrollers by Tim Wilmshurst

[3]. Igarashi, G., Saeki, S., Takahata, N., Sumikawa, K., Tasaka, S., Sasaki, Y., Takahashi, M., Sano ,Y. (1995). Ground-water radon anomaly before the Kobe earthquake in Japan Science Vol. 269.

[4]. Nazaroff, W.W.; Nero, A.V., Radon and its decay products in indoor air, 1988, United States

[5]. Advanced PIC Microcontroller Projects in C by Dogan Ibrahim

[6]. Serial Communications by David Benson

[7]. PIC Microcontroller and Embedded systems: Muhammad Ali Mazidi, Rolind D. Mckinlay \& Danny Causey

[8]. Designing Embedded Systems with PIC Microcontrollers by Tim Wilmshurst

[9]. Samuel R. Madden, Robert Szewczyk, Michael J. Franklin, and David Culler. Supporting aggregate queries over ad-hoc wireless sensor networks. In Workshop on Mobile Computing and Systems Applications, 2002.

[10]. Digital principles and applications D.P.Leach, A.P.Malvino and G. Saha. $6^{\text {th }}$ edition, the McGraw-Hill.

[11]. 'ICRTITCS 2012' - International Journal of Embedded Systems and Applications, 'INTERFACING OF LCD USING PIC18. 